\title{
MicroRNAs as potential biomarkers for diagnosis, therapy and prognosis of gastric cancer
}

This article was published in the following Dove Press journal:

OncoTargets and Therapy

\author{
Hai-Liang Yuan \\ Ting Wang \\ Kun-He Zhang
}

Department of Gastroenterology, the First Affiliated Hospital of Nanchang University, Jiangxi Institute of Gastroenterology \& Hepatology, Nanchang, People's Republic of China
Correspondence: Kun-He Zhang Department of Gastroenterology, the First Affiliated Hospital, Nanchang University, 17 Yongwai Zheng Street, Nanchang, 330006, People's Republic of China

Tel +86 79l 88692357

Email khzhang@ncu.edu.cn

\begin{abstract}
Despite the widespread use of endoscopy and conventional tumor biomarkers, gastric cancer (GC) remains one of the most frequent causes of cancer-related deaths worldwide due to its late diagnosis and poor response to treatment. Valuable and practical biomarkers are urgently needed to screen patients with a high risk of GC that can complement endoscopic diagnosis. Such biomarkers will enable the efficient prediction of therapeutic response and prognosis of GC patients and favor the establishment of an effective treatment strategy for each and every patient. MicroRNAs (miRNAs) are a class of small non-coding RNA sequences that play important roles in modulating key biological processes by regulating the expression of target genes. Expectedly, miRNAs are abnormally expressed within the tumor tissue and in associated biological fluids of GC patients including their blood, gastric juice, and urine. Accumulating evidence indicates that miRNAs are potential biomarkers with multiple diagnostic functions for GC. Here, we review recent advances and challenges in using miRNAs, particularly biofluid miRNAs, as GC biomarkers with potential clinical applications including diagnosing, clinically staging, and predicting malignant behaviors, therapy response, recurrence after surgery and survival time.

Keywords: gastric cancer, microRNA, biomarker, diagnosis, prognosis
\end{abstract}

\section{Introduction}

Gastric cancer (GC) is one of the most frequent causes of death in cancer patients and a major public health problem worldwide. ${ }^{1}$ Nearly $95 \%$ of GCs are adenocarcinomas which are classified into three histological types: diffuse, intestinal, and hybrid gastric cancers according to the Lauren system, ${ }^{2}$ and cardia and non-cardia gastric cancers according to the World Health Organization. ${ }^{3}$ Although surgery, chemotherapy, and other therapies continuously progress in the management of GC patients, their prognosis remains poor. ${ }^{4}$ An early and precise diagnosis is essential for improving the survival rate of patients with GC.

Endoscopy followed by biopsy is a common tool for detecting GC and remains the most accurate method for GC diagnosis. ${ }^{5}$ However, this procedure is invasive and expensive, and it may cause the patients discomfort and fear. In addition, asymptomatic patients generally do not receive an endoscopic examination. ${ }^{6}$ Conventional serum tumor biomarkers, such as carcino-embryonic antigen (CEA), carbohydrate antigen 19-9 (CA19-9), carbohydrate antigen 72-4 (CA72-4), and carbohydrate antigen 50 (CA50), are frequently used for gastrointestinal tumor detection but are not recommended for GC due to a lack of sensitivity and/or specificity. ${ }^{7,8}$ Thus, simple, costeffective, and noninvasive methods are urgently required for GC detection.

MicroRNAs (miRNAs; miRs) are non-coding RNA molecules that regulate eukaryotic gene expression at the post-transcriptional level in humans, animals, 
and plants, and these molecules are abnormally expressed frequently in cancer tissues. MiRNAs can be delivered from tumor cells into biofluids by exosome particles or microvesicles, which protect miRNAs from the degradation by RNases and have been found in serum, plasma, urine, tear, and gastric juice. ${ }^{9}$ There are accumulating evidences that miRNAs can be potential biomarkers for various cancer types. ${ }^{10}$ Several miRNAs are abnormally expressed in the tumor tissues and body fluids of GC patients and show promise in clinical application. ${ }^{11}$

In this review, we summarize and discuss the recent advances and challenges in the use of miRNAs, particularly miRNAs in the biological fluids, as potential GC biomarkers for screening and diagnosing, clinically staging, and predicting malignant behaviors, therapy response, recurrence after operation, and survival time.

\section{MicroRNAs are emerging biomarkers for cancer MicroRNAs and biofluid microRNAs}

MiRNAs were discovered more than 20 years ago but did not receive attention until 2001. ${ }^{12,13}$ To date, thousands of human miRNAs have been identified. Most human genes are directly targeted by miRNAs. ${ }^{14}$ MiRNAs play an important role in the regulation of almost all aspects of biology, including the pathogenesis of cancer. ${ }^{15}$ MiRNAs affect cancer progression by regulating cancer genes (tumor suppressor miRNA) or tumor suppressor genes. ${ }^{16}$ Additionally, miRNAs can regulate different levels of gene expression (transcription and translation) and serve as tumor suppressors or oncogenes. ${ }^{17}$

Biofluid miRNAs, also known as circulating miRNAs, are a type of miRNA with stability in body fluids. These molecules are secreted into body fluids by tumor cells via specific pathways. ${ }^{18}$ MiRNAs can be secreted through exosomes. ${ }^{19}$ Another pathway is passive secretion after cell necrosis and apoptosis. ${ }^{20}$ The most widely studied miRNAs are those encapsulated in exosomes. The amount of miRNAs secreted by exosomes was inconsistent with the miRNAs in the exosomes, ${ }^{21}$ indicating that miRNAs are selectively packed into exosomes. However, the mechanism by which miRNAs are selectively secreted from these vesicles remains unknown. Interestingly, a recent study revealed that some of the highly expressed serum miRNAs in patients with GC might be derived not from GC tissues but other non-cancerous tissues. ${ }^{22}$ Therefore, the origin of circulating miRNAs needs to be further elucidated.

Both tissue and biofluid miRNAs are abnormally expressed in many diseases, especially in tumors. The expression levels of miRNAs in cancer tissues are related to the tumor status, size, invasion, and metastasis and may be used for early cancer detection. ${ }^{23}$ However, the miRNAs in tumor tissues are not ideal candidates for clinical diagnostic application, as obtaining tissue samples involves an invasive procedure. In 2008, a study reported that miRNAs in the blood of tumor patients can be used as biomarkers for cancer, ${ }^{24}$ and they have recently been considered promising biomarker candidates for cancer. ${ }^{25}$ Compared with intracellular miRNAs, biofluid miRNAs are non-invasive biomarkers for clinical application in cancer. For example, serum miR-30e and miR-223 can be used to distinguish hepatocellular carcinoma from healthy controls, ${ }^{26}$ the expression of serum miR-365 was related to the overall survival of patients with lung cancer; ${ }^{27}$ and the serum expression levels of miR-21, miR-17 and miR-92 were highly correlated with recurrence after adjuvant chemotherapy in patients with colon cancer. ${ }^{28}$

\section{MicroRNAs in gastric cancer}

MiRNAs play important roles in the molecular and cellular biology of GC, including proliferation, migration and invasion. ${ }^{29}$ Studies have revealed that miR-17-5p could serve as an oncogene (oncomiR) to promote cell growth and invasion by regulating transforming growth factor-beta receptor 2 in GC. ${ }^{30}$ In contrast, miR-203 is a tumor suppressor that is capable of inhibiting tumor cell proliferation, migration, and invasion by regulating the levels of Slug in GC. ${ }^{31}$ Ye et $\mathrm{al}^{32}$ recently demonstrated that miR-647 could serve as a tumor suppressor to inhibit GC metastasis by targeting the SRF/ MYH9 axis.

Interestingly, the miRNAs that are expressed in GC may also serve as tumor suppressors and oncogenes. For example, miR-10b in GC could promote tumor cell invasion via the up-regulation of RhoC and AKT phosphorylation through targeting HOXD $10,{ }^{33}$ and could also dramatically suppress cell proliferation, migration, and invasion and induce apoptosis. ${ }^{34}$ Several studies have shown that miR-107 could act as an oncogene to promote the invasion and metastasis of GC through a variety of targets (including DICER1 and NF1), ${ }^{35,36}$ but Feng et $\mathrm{al}^{37}$ found that miR-107 was a tumor suppressor that inhibited the proliferation and invasion of gastric cancer cells. The dual effects of these miRNAs may be related to the fact that a single miRNA can target multiple genes and that a single gene can be regulated by multiple miRNAs. ${ }^{38}$ The mechanism underlying the complex interaction between miRNAs and targets remains unclear in cancer. ${ }^{39}$ 


\section{MiRNAs as diagnostic biomarkers for gastric cancer}

MiRNAs are abnormally expressed in the tumor tissues and body fluids of GC patients. Because the detection of miRNAs in plasma, serum, urine, and gastric juice can be used in diagnosis, several studies have been conducted to evaluate the diagnostic performance of miRNAs in body fluids for GC, and the results have shown that many miRNAs have certain value for $\mathrm{GC}$ in diagnosis, recurrence monitoring and/or clinical staging.

\section{MiRNAs for the detection of gastric cancer \\ MiRNAs in plasma/serum}

Tsujiura et $\mathrm{al}^{40}$ analyzed the levels of miRNAs in the plasma of 69 patients with GC and found that some miRNAs, such as miR-17-5p, miR-21, miR-106a and miR-106b, are up-regulated. Zhang et $\mathrm{al}^{41}$ showed that miR-16-5p and miR-19b-3p are consistently elevated in the plasma of GC patients compared with controls and these molecules had potential diagnostic value for GC, with areas under the receiver operating characteristic curves (AUROCs) of 0.772 and 0.749 , sensitivities of $49.0 \%$ and $81.3 \%$, and specificities of $91.0 \%$ and $58.6 \%$, respectively. Tsai et $\mathrm{al}^{42}$ investigated the expression levels of miR-196a/b in plasma and showed a much higher specificity for the diagnosis of GC than CEA and CA19-9, with sensitivity and specificity of $69.5 \%$ and $97.6 \%$ for miR-196a and $62.2 \%$ and $96.1 \%$ for miR-196b, respectively. Hung et al ${ }^{43}$ found that compared with healthy controls, miR-376c was up-regulated in the tissue, plasma, and urine of GC patients and the levels of miR-376c in plasma exhibited a diagnostic value for GC, with AUROC of 0.77, sensitivity of $71.0 \%$ and specificity of $78.0 \%$. Another study reported that the miR-206 levels in serum were lower in GC patients $(\mathrm{n}=150)$ than in healthy controls $(\mathrm{n}=150)$, with AUROC of 0.861 , sensitivity of $78 \%$ and specificity of $86 \%$ for GC detection. ${ }^{44}$

The performance of single miRNAs in blood is not ideal for GC diagnosis because of tumor heterogeneity. Thus, a combination of plasma miRNAs may help improve their diagnostic performance for GC. Zhu et a ${ }^{45}$ conducted a fourphase study on the diagnostic value of circulating miRNAs in 124 patients with gastric non-cardia adenocarcinoma (GNCA), 36 patients with gastric cardia adenocarcinoma (GCA) and 160 cancer-free controls. These authors found that the levels of five miRNAs (miR-16, miR-25, miR-92a, miR-451 and miR-486-5p) were consistently elevated in the plasma of GC patients and could be used as potential markers for diagnosing GNCA, with AUROCs of 0.850 to 0.925 in the training set and 0.694 to 0.790 in the validation phases, respectively. The panel of the five miRNAs presented a high diagnostic accuracy for early-stage GNCA, with AUCs of 0.989 and 0.812 in the training and validation phases, respectively. However, in another report, the six serum miRNAs (miR10b-5p, miR132-3p, miR185-5p, miR195-5p, miR-20a3p, and miR296-5p) used as a biomarker panel for discriminating GC from normal controls were not as good, exhibiting AUROCs of 0.764 and 0.702 in the training and validation phases, respectively. ${ }^{46}$ The different results between the two groups may be due to different miRNAs used in the panels. Additionally, various analysis platforms for miRNA detection may also result in different results. ${ }^{47}$ However, miRNAs in combination may be crucial for improving diagnostic performance.

\section{MiRNAs in gastric juice}

Chen et $a{ }^{10}$ examined the stability of miRNAs under harsh conditions, including boiling, low/high $\mathrm{pH}$ and freeze-thaw cycles, and the results showed that miRNAs still remained stable after treatment for $3 \mathrm{~h}$ in low $(\mathrm{pH}=1)$ or high $(\mathrm{pH}=13)$ $\mathrm{pH}$ solution, indicating that miRNAs in gastric juice might be used as novel biomarkers for GC.

Several studies have shown that the presence of miR-21, miR-106a, miR-133a, miR-421, miR-129-1-3p, miR-129$2-3 p$ and miR-129 in gastric juice could be good candidates for GC diagnosis, even in early GC. Cui et $\mathrm{al}^{48}$ found that miRNAs in gastric juice were valuable for discriminating GC from benign gastric diseases, with AUROCs, sensitivities and specificities of $0.969,85.7 \%$ and $97.8 \%$ for miR-21 and $0.871,73.8 \%$ and $89.3 \%$ for miR-106a, respectively; and an AUROC up to 0.982 was observed for the combination of miR-21 and miR-106a. Shao et $\mathrm{a}^{49}$ found that the miR-133a levels in gastric juice were dramatically lower in GC patients than in both healthy subjects and individuals with benign gastric diseases, with AUROC of 0.907, sensitivity of $85.9 \%$ and specificity of $84.8 \%$ for discriminating $\mathrm{GC}$ from gastric benign diseases. Zhang et a $\mathrm{l}^{50}$ revealed that the levels of miR-421 in gastric juice were lower in patients with GC than in patients with gastric benign diseases, which distinguished GC from gastric benign patients with AUROC of 0.767 , sensitivity of $71.4 \%$, and specificity of $71.7 \%$; they found the same positive rates in early and late gastric cancer $[71.4 \%(5 / 7)$ vs. $71.4 \%(25 / 35)]$. In contrast, the positive rates in early and late gastric cancer patients were, respectively, $14.3 \%(1 / 7)$ and $40.0 \%(14 / 35)$ for serum CEA and $42.8 \%(3 / 7)$ and $74.3 \%$ (26/35) for gastric juice CEA. 
The expression levels of miR-129-1-3p and miR-129-2-3p in gastric juice were also lower in GC patients than in healthy subjects, with AUROCs of 0.639 for miR-129-1-3p and 0.651 for miR-129-2-3p. ${ }^{51}$

Compared with blood, the collection of gastric juice from the patients through gastroscopy or the insertion of a nasogastric tube is invasive. Thus, efforts are needed to develop non-invasive methods for collecting gastric juices in the future.

\section{MiRNAs in urine}

The urinary miR-376c also exhibited diagnostic value in GC patients, with AUROC of 0.7 , sensitivity of $60.0 \%$ and specificity of $64.0 \%{ }^{43}$ A recent study showed that miR-21-5p was highly expressed in urine of gastric cancer patients compared with healthy controls and was significantly reduced after surgical resection of gastric cancer. ${ }^{52}$ Because the collection of urine is convenient and completely non-invasive, additional studies on using urinary miRNAs as biomarkers for GC diagnosis are warranted.

\section{MiRNAs in exosome}

Exosomes are lipid bilayer membrane-enclosed small vesicles in the extracellular environment, which are secreted by cells and contain a variety of molecules, including miRNAs. Tumor cells secrete excessive amount of exosomes related to tumor biological behaviors. miRNAs in exosomes are protected from ribonuclease degradation. ${ }^{53}$ Therefore, they are stable in body fluid and suitable as GC biomarkers. Ma et $\mathrm{al}^{54}$ found that miR-221 in exosomes from the peripheral blood was highly expressed ( 2.5 fold) in GC patients compared with healthy controls and significantly reduced after surgical resection. Exosomal miR-106a-5p and miR-19b-3p in plasma were dramatically elevated in GC patients compared with controls, with AUROCs of 0.786 for miR-106a-5p, 0.769 for miR-19b-3p, and 0.814 for both miRNAs in combination in the diagnosis of GC. ${ }^{55}$ However, more studies are required to evaluate the diagnostic value of exosomal miRNAs for GC.

\section{MiRNAs for monitoring the recurrence of gastric cancer after resection}

Biofluid miRNAs are valuable for monitoring the recurrence of gastric cancer after operation. Hou et $\mathrm{al}^{44}$ detected the serum miRNA-206 levels in pre-operative $(n=150)$, post-operative $(\mathrm{n}=150)$, and recurrent $(\mathrm{n}=110)$ patients with $\mathrm{GC}$ and showed that miR-206 expression in serum was significantly up-regulated in paired post-operative samples and reduced in recurrent samples, with AUROC of 0.85 for discriminating recurred and non-recurred patients. Liu et $\mathrm{al}^{56}$ screened a large number of plasma miRNAs in 407 patients with GC at stages II and III and created a 7-miRNA classifier and an index with $7 \mathrm{miRs}$ and pathological factors to predict the 3-year post-operative recurrence; the results showed that the AUROCs of the classifier and the index were, respectively, 0.725 and 0.841 in the training set and 0.627 and 0.771 in the validation set. The high-risk patients determined by the signatures had a significantly shorter disease-free survival and overall survival time than did the low-risk patients.

Tissue miRNAs also have potential value for diagnosing the recurrence of gastric cancer after resection. Zhang et $\mathrm{al}^{57}$ detected hsa-miR-375 and hsa-miR-142-5p in 34 frozen fresh tissues and 38 formalin-fixed paraffin-embedded tissues and combined these two miRNAs as a classifier to predict GC recurrence, which showed AUROCs of 0.75 and 1.00 in the training and validation phases, respectively. Similarly, Park et $\mathrm{al}^{58}$ conducted a study using 50 paraffin-embedded tissues and found that hsa-miR-21-5p was significantly higher in recurrence group than in the group without recurrence, with AUROC of 0.723 , specificity of $65.5 \%$ and sensitivity of $86.7 \%$.

MiRNAs can be used to predict recurrence in early gastric cancer after endoscopic submucosal dissection (ESD). Xue et $\mathrm{al}^{59}$ detected the expression levels of miR-328 in the early gastric cancer tissues from ESD and found significantly lower levels in the recurrence group $(\mathrm{N}=96)$ than in the non-recurrence group $(\mathrm{N}=134)$. They also noted that the recurrence rate was significantly lower in the group with higher levels of miR-328 than in the group with lower levels of miR-328.

\section{MiRNAs for gastric cancer staging}

The TNM system is the most widely used cancer staging system, and it is based mainly on the primary tumor size (T), the extent of lymph node involvement $(\mathrm{N})$ and the presence of distant metastasis (M). In gastric cancer tissues, the expression of miRNAs, including microRNA-630, microRNA-196a/196b and microRNA-29, was significantly related to the TNM staging system. ${ }^{60-62}$

Biofluid miRNAs are also associated with tumor staging in gastric cancer. Zhang et $\mathrm{al}^{41}$ found that the plasma miR-16-5p and plasma miR-19b-3p showed good performance in differential diagnosis of GC cases with different TNM stages (IV stage: AUC $=0.832$ for miR-16-5p; III stage: $\mathrm{AUC}=0.822$ for miR-19b-3p); these authors also showed that the diagnostic performances of the two miRNAs improved 
when gastric cancer progressed from earlier stages to advanced stages.

\section{MiRNAs as prognostic biomarkers for gastric cancer}

Accumulating studies have shown that some biofluid miRNAs are capable of predicting the survival of GC patients. An earlier study found that the expression levels of plasma miR-21 were related to the overall survival (OS) of GC patients: the 3-year survival rate was lower in patients with higher miR-21 levels than in patients with lower levels $(64.9 \%$ vs $85.9 \%) .{ }^{63}$ In a recent study, Zhuang et al ${ }^{64}$ found that the plasma miR-23b levels were higher in GC patients $(n=138)$ than in normal subjects $(n=50)$ and were remarkably related to the survival of GC patients: the 5-year OS was much lower in GC patients with higher miR-23b levels than in patients with lower levels (29.4\% vs 75.7\%).

MiRNAs in GC tissues are also related to the OS of GC patients. Yang et $\mathrm{al}^{65}$ reported that the miR-206 expression levels were negatively correlated with the survival rates of GC patients: 1, 3, and 5-year survival rates were $76.9 \%$ (40/52), 69.2\% (36/52) and 57.7\% (30/52), respectively, in patients with higher levels of miR-206, but $91.3 \%$ (42/46), $84.8 \%(39 / 46)$, and 80.6\% (37/46), respectively, in patients with lower levels. These findings were further confirmed by Shi et al. ${ }^{66}$ The expression of miR-92a in GC tissue was also significantly related to OS of GC patients: GC patients with higher levels of miR-92a had shorter survival times than those with lower levels of miR-92a in stage II (OS 30.6 vs 64.9 months) and stage III (OS 29.7 vs 58.9 months). ${ }^{67}$

A recent meta-analysis demonstrated that many miRNAs, such as miR-20b, miR-141, miR-145 and miR-486-5p, were associated with survival prediction in patients with gastric cancer. ${ }^{68}$

Recently, Ding et al $^{69}$ developed an eight-miRNA prognostic model (miR-145, miR-184, miR-20b, miR-9-1, miR-9-2, miR-1537, miR-549 and miR-802) for predicting the survival of stomach adenocarcinoma patients, and the results showed that patients with high scores had a poor OS (median OS: 1,811 days vs 562 days), similar to the finding for earlystage patients (median OS: 2,197 days vs 652 days).

\section{MiRNAs as biomarkers for histological behaviors of gastric cancer Invasion}

Some miRNAs are involved in the activation of GC invasion. The overexpression of miR-148a inhibited GC cell migration and invasion in vitro and metastasis formation in vivo by targeting ROCK $1 .{ }^{70}$ Interestingly, miR-148a also targets matrix metalloproteinase (MMP) 7, which may lead to the invasion of GC. ${ }^{71}$ In a recent study, Qiu et al $^{72}$ found that both miR-148a and miR-26a were down-regulated in tumor tissues and that the up-regulated expression of miR-26 inhibited the proliferation, migration, invasion, and colony formation of MGC-803 cells. Similarly, the up-regulation of miR-153 in $\mathrm{MKN}-45$ cells reduced cell migration and invasion, whereas the down-regulation of miR-153 in SGC-7901 cells promoted cell migration and invasion. ${ }^{73}$

\section{Metastasis}

MiRNAs are related to GC metastasis. The overexpression of some miRNAs in GC tissues is significantly associated with lymph node metastases, such as miR-196a/b, miR-10a, miR-23a/b, ${ }^{61,74,75}$ and some miRNAs with low expression in GC tissues are also related to lymph node metastasis. miR-125a-3p was down-regulated in GC tissues and associated with lymph node and liver metastasis. ${ }^{76}$ Zhang et al ${ }^{77}$ found that the expression levels of miR-145-5p were significantly lower in GC tissues than that in adjacent normal tissues, which was associated with lymph node metastasis, metastasis stage, and distant metastasis. Recently, Li et $\mathrm{al}^{78}$ observed the expression levels of miR-30c-2-3p and miR133a-3p in GC tissues from 338 patients with metastases and 23 patients without metastases and found that the levels of these miRNAs were related to lymph node metastasis.

The expression levels of biofluid miRNAs are also associated with GC metastasis. Yang et $\mathrm{al}^{79}$ found that the serum miR-20a levels were correlated with lymph node metastasis in GC. The levels of serum miR-203 were dramatically lower in GC patients $(n=130)$ than in healthy controls, with odds ratios of 9.86 (95\% CI 2.73-26.59) for lymph node metastasis and 12.04 (95\% CI 2.07-230.0) for distant metastasis. ${ }^{80}$

\section{Histological type}

Stomach adenocarcinoma is divided into three histological types: intestinal, diffuse and undifferentiated, ${ }^{2}$ and each type has different clinical features and biological behaviors, particularly intestinal type and diffuse type ${ }^{81}$ In the mouse model of diffuse-type gastric cancer (DGC), four miRNAs (miR-103, miR-107, miR-194, and miR-210) were highly expressed in the serum and cancer tissues compared with the corresponding controls, and when these four miRNAs in serum were used as biomarkers for the early detection of DGC, they showed high AUROCs, sensitivities and 
specificities: $0.881,81.8 \%$ and $95.7 \%$ for miR-103, 0.909 , $90.9 \%$ and $95.7 \%$ for miR-107, $0.925,90.9 \%$ and $95.7 \%$ for miR-194, and $0.846,72.7 \%$ and $87.0 \%$ for miR-210, respectively. ${ }^{82}$ Azarbarzin et $\mathrm{al}^{83}$ showed that the miR-383 levels were down-regulated in intestinal-type gastric adenocarcinoma compared with non-tumor tissues; this feature was able to distinguish intestinal gastric cancer patients from healthy controls, with a sensitivity of $76 \%$ and specificity of $84 \%$.

\section{MiRNAs as therapeutic biomarkers for gastric cancer}

Studies in cellular biology have shown that the levels of the miRNAs that are expressed in GC cells are associated with the sensitivity to anticancer drugs. The expression levels of miR-15b or miR-16 could attenuate multidrug resistance in GC cells by targeting the BCL2 gene: the apoptotic cells were higher than $30 \%$ in the overexpression group but approximately $10 \%$ in the control group. ${ }^{84}$ MiR-185 shows low levels in gastric cancer cell lines and clinical tissues, and its overexpression increased the sensitivity of GC cells to anticancer drugs by targeting ARC (the apoptotic GC cells were approximately $40 \%$ in the miR-185 overexpression group and $15 \%$ in the control group). ${ }^{85} \mathrm{MiR}-31$, as a tumor suppressor involved in GC tumorigenesis, increased the sensitivity to chemotherapy (5-fluorouracil) in MKN-45 cells compared to the control groups. ${ }^{86}$

In clinical studies, miRNAs were associated with chemosensitivity or chemoresistance. Kim et $\mathrm{al}^{87}$ analyzed the association of the levels of miRNAs in the biopsy tissues with time to progression (TTP) of disease after chemotherapy in 90 patients with GC and found that 30 miRNAs were inversely correlated and 28 miRNAs were positively correlated with TTP in 82 cancer patients, including six up-regulated miRNAs (let-7g, miR-342, miR-16, miR-181, miR-1, and miR-34) related to chemosensitivity and another six up-regulated miRNAs (miR-518f, miR-520a, miR-520d, miR-519e, miR-363, and miR-517) related to chemoresistance. By using tissue samples from 54 patients who underwent palliative chemotherapy, Smid et $\mathrm{al}^{88}$ found that the miRNAs with high levels of expression (miR-150, miR342-3p, miR-181b, miR-221, and miR-224) and the miRNA with a low expression level (miR-520h) were related to TTP and OS in GC patients, indicating that these miRNAs could be used as biomarkers to predict the effectiveness of treatment. Shang et $\mathrm{al}^{89}$ found that the overexpression of miR-27b was sufficient to sensitize GC cells to chemotherapy in vitro and in vivo and that the expression levels of miR-27b and
miR-508-5p were associated with chemosensitivity and overall survival in 114 patients with GC.

MiRNAs may predict the outcome of GC target therapy. Sui et $\mathrm{al}^{90}$ analyzed the association of tissue miR-125b with the outcome of 28 patients with GC who were treated with trastuzumab (a targeted drug used for HER2-positive cancer); they found that the overall survival rate was dramatically lower in the patients with high miR-125b expression than those with low miR-125b expression, suggesting that miRNAs could be used as biomarkers to more precisely predict the efficiency of tumor target therapy.

Surgically treated GC patients who had high levels of serum miR-203 showed longer overall survival time than those with low levels of serum miR-203, and the serum miR-203 level was an independent prognostic biomarker. ${ }^{80}$ Similarly, the serum miR-204 level was a biomarker for predicting prognosis in GC patients with systemic treatment. ${ }^{91}$ A recent study showed that miR-21-5p was highly expressed in the urine of gastric cancer patients compared with healthy controls and noted that its expression was reduced following surgery, suggesting that urine miR-21-5p can be used as a valuable noninvasive biomarker to evaluate the prognosis of GC patients undergoing surgical treatment. ${ }^{52}$

\section{MicroRNAs as multifunctional biomarkers for gastric cancer}

Interestingly, many miRNAs, such as miR-16-5p, miR-19b-3p, miR-196a/b, miR-376c, miR-206, miR-21-5p, miR-18a and miR-144, have multiple diagnostic potential (Figure 1) for GC. ${ }^{41-44,52,92-94}$ These miRNAs have predictive values not only in diagnosis but also in evaluating prognosis and/or treatment responses in patients with GC.

Su et $\mathrm{al}^{92}$ reported that plasma miR-18a was significantly up-regulated in GC patients compared with controls and had potential diagnostic value for GC, with AUROC of 0.907 , specificity of $84.6 \%$ and sensitivity of $80.5 \%$. This miRNA was also associated with the prognosis of GC patients: the 5-year OS was much worse in GC patients with higher levels of plasma miR-18a than in patients with lower levels of plasma miR-18a. Moreover, the expression of miR-18a was also significantly associated with pathological grades and lymph metastasis. Tsujiura et al reported similar results. ${ }^{93}$

MiR-144 is another multifunctional miRNA in GC. Liu et $\mathrm{al}^{94}$ found that miR-144 was significantly down-regulated in the plasma of GC patients, with AUROC of 0.821 , specificity of $83.6 \%$ and sensitivity of $71.5 \%$ for diagnosing $\mathrm{GC}$, and the miR-144 levels in both tissue and serum were associated with the prognosis of GC patients following 


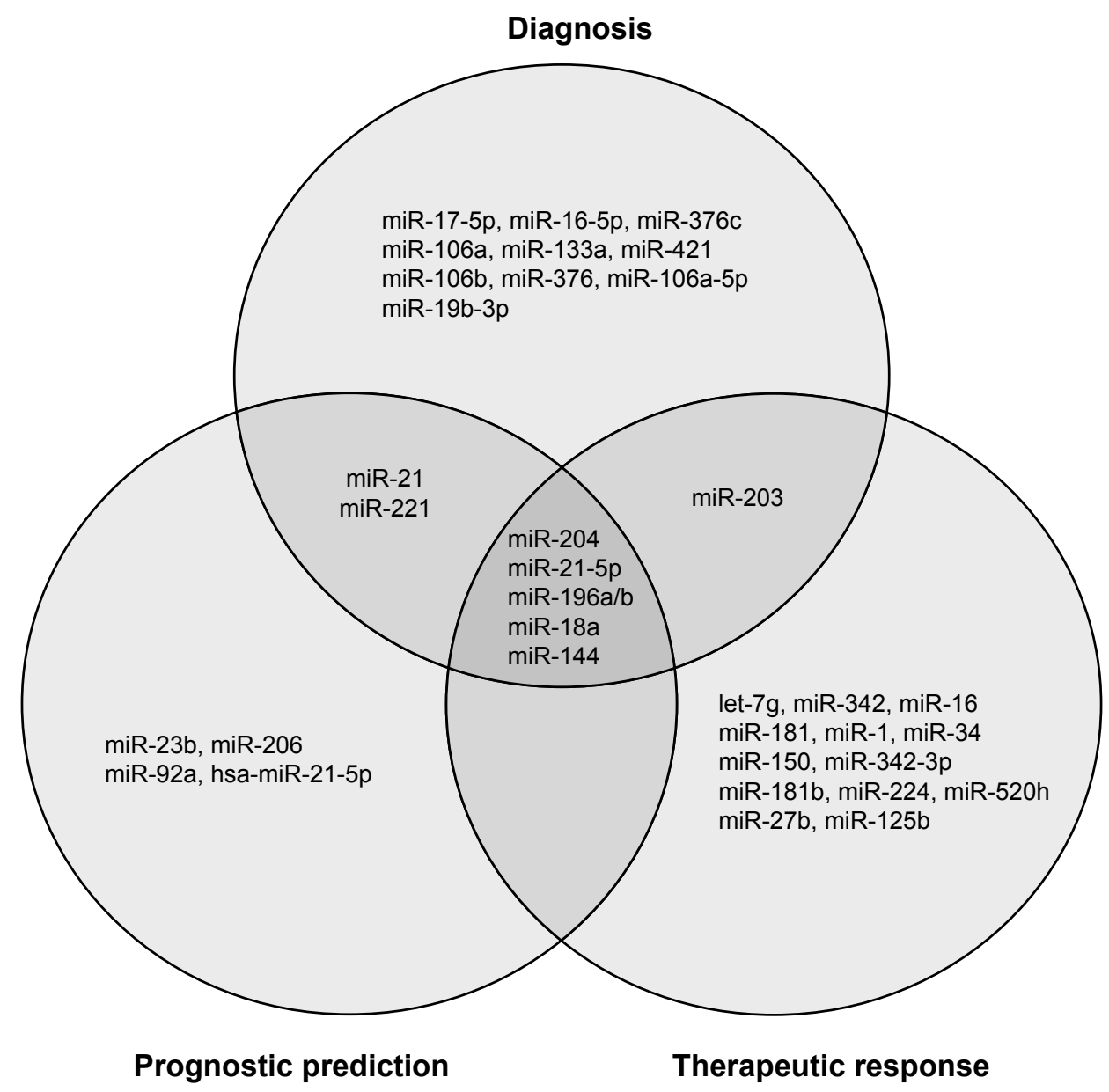

Figure I The clinical function sorting of miRNAs as biomarkers of gastric cancer.

surgery: the 5-year OS was much lower in GC patients with lower tissue miR-144 levels than that in patients with higher levels (26.1\% vs $76.0 \%$ ); similarly, the 5-year OS was much worse in GC patients with lower serum miR-144 levels than that in patients with higher levels. These findings suggest that miR-144 can serve as a multifunctional biomarker for patients with gastric cancer, which has a predictive value for diagnosis, treatment, and prognosis.

\section{Challenges of miRNAs as clinical biomarkers for gastric cancer}

Although miRNAs hold promise as GC biomarkers, some challenges should be overcome before these molecules are used as clinical tests for GC. The first issue is to validate the diagnostic value of miRNAs. The reliability of using miRNAs as biomarkers for the diagnosis, prognosis, and treatment response of gastric cancer should be confirmed via further larger-scale studies. The current reports are generally single-center studies with small samples. The second issue is the sensitivity and specificity of miRNAs to GC.
The identification of sensitive miRNAs that can be used as biomarkers may be a challenge due to the heterogeneity of GC itself. In recent reports, few miRNAs in GC are highly sensitive and ideal for clinical application. Similarly, the specificity of miRNAs to GC is also an issue due to the "universality" of the miRNAs themselves. For example, the high expression of miR-21 was related to poor prognosis not only in GC patients ${ }^{63}$ but also in patients with other cancers, including hepatocellular carcinoma and breast cancer. ${ }^{95,96}$ The third issue is the methodology for miRNA detection. The biological specimens used for GC detection include tissue, plasma and serum, urine and gastric juice. For each type of specimen that is utilized in miRNA analysis, a standardized procedure is needed before miRNAs as biomarkers are translated into clinical application, including specimen collection, pre-analytical preparation, and analysis. ${ }^{97,98}$ The fourth issue is the further realization of miRNA functions. There is an enormous number of miRNAs in the human body. The fact that a single miRNA can target multiple genes and that a single gene can be targeted by multiple 
miRNAs indicates a regulation network of miRNAs in the body. Thus, substantial studies on functions and mechanism of miRNAs in GC are crucial to facilitate the application of miRNAs in the clinic.

\section{Conclusions}

Classic tumor biomarkers, such as CEA, CA72-4, and CA19-9, are poor in sensitivity and specificity for GC diagnosis. ${ }^{7,8}$ Accumulating evidence indicates that miRNAs possess great promise as tumor biomarkers. The abnormal expression of miRNAs can be detected in patients with GC not only in tissue samples but also in body fluids, including plasma/serum, urine, and gastric juice. The miRNAs in biofluids are stable even under harsh conditions, and these molecules are ideal clinical biomarkers due to the non-invasive nature of their sample collection. The expression levels of many miRNAs in the tissues or biofluids of GC patients are valuable for diagnosis, prediction of prognosis and response to therapy, and/or monitoring recurrence after resection, and these molecules may be "multifunctional" biomarkers; ie, a single miRNA has predictive value in diagnosis, therapy response, and prognosis simultaneously. However, some obstacles should be overcome for miRNAs to translate into the clinic, including the validation of diagnostic value in larger-scale studies, the identification of more sensitive and specific miRNAs to gastric cancer, the development of standardized methods for miRNA detection and analysis, and more knowledge of miRNA biology. After overcoming these challenges, the use of miRNAs as multifunctional biomarkers for the precise management of GC may be realized.

\section{Acknowledgments}

This work was supported by the National Natural Science Foundation of China (No 81560479) and the Science and Technology Project of the Education Department of Jiangxi Province (No KJLD13014).

\section{Disclosure}

The authors report no conflicts of interest in this work.

\section{References}

1. Ferlay J, Soerjomataram I, Dikshit R, et al. Cancer incidence and mortality worldwide: sources, methods and major patterns in GLOBOCAN 2012. Int J Cancer. 2015;136(5):E359-E386.

2. Lauren $P$. The two histological main types of gastric carcinoma: diffuse and so-called intestinal-type carcinoma. An attempt at a histo-clinical classification. Acta Pathol Microbiol Scand. 1965;64: 31-49.

3. Colquhoun A, Arnold M, Ferlay J, Goodman KJ, Forman D, Soerjomataram I. Global patterns of cardia and non-cardia gastric cancer incidence in 2012. Gut. 2015;64(12):1881-1888.
4. Newton AD, Datta J, Loaiza-Bonilla A, Karakousis GC, Roses RE. Neoadjuvant therapy for gastric cancer: current evidence and future directions. J Gastrointest Oncol. 2015;6(5):534-543.

5. Veitch AM, Uedo N, Yao K, East JE. Optimizing early upper gastrointestinal cancer detection at endoscopy. Nat Rev Gastroenterol Hepatol. 2015;12(11):660-667.

6. Choi J, Kim SG, Im JP, Kim JS, Jung HC, Song IS. Comparison of endoscopic ultrasonography and conventional endoscopy for prediction of depth of tumor invasion in early gastric cancer. Endoscopy. 2010;42:705-713.

7. Shimada H, Noie T, Ohashi M, Oba K, Takahashi Y. Clinical significance of serum tumor markers for gastric cancer: a systematic review of literature by the Task Force of the Japanese Gastric Cancer Association. Gastric Cancer. 2014;17(1):26-33.

8. Liang $\mathrm{Y}$, Wang W, Fang $\mathrm{C}$, et al. Clinical significance and diagnostic value of serum CEA, CA19-9 and CA72-4 in patients with gastric cancer. Oncotarget. 2016;7(31):49565-49573.

9. Gilad S, Meiri E, Yogev Y, et al. Serum microRNAs are promising novel biomarkers. PLoS One. 2008;3(9):e3148.

10. Chen X, Ba Y, Ma L, et al. Characterization of microRNAs in serum: a novel class of biomarkers for diagnosis of cancer and other diseases. Cell Res. 2008;18(10):997-1006.

11. Wang QX, Zhu YQ, Zhang H, Xiao J. Altered MiRNA expression in gastric cancer: a systematic review and meta-analysis. Cell Physiol Biochem. 2015;35(3):933-944.

12. Lee RC, Feinbaum RL, Ambros V. The C. elegans heterochronic gene lin-4 encodes small RNAs with antisense complementarity to lin-14. Cell. 1993;75(5):843-854.

13. Lee RC, Ambros V. An extensive class of small RNAs in Caenorhabditis elegans. Science. 2001;294(5543):862-864.

14. Friedman RC, Farh KK, Burge CB, Bartel DP. Most mammalian mRNAs are conserved targets of microRNAs. Genome Res. 2009;19(1): 92-105.

15. Kim VN. MicroRNA biogenesis: coordinated cropping and dicing. Nat Rev Mol Cell Biol. 2005;6(5):376-385.

16. Svoronos AA, Engelman DM, Slack FJ. OncomiR or tumor suppressor? The duplicity of microRNAs in cancer. Cancer Res. 2016;76(13): 3666-3670.

17. Zhang Z, Li Z, Li Y, et al. MicroRNA and signaling pathways in gastric cancer. Cancer Gene Ther. 2014;21(8):305-316.

18. Arroyo JD, Chevillet JR, Kroh EM, et al. Argonaute2 complexes carry a population of circulating microRNAs independent of vesicles in human plasma. Proc Natl Acad Sci U S A. 2011;108(12):5003-5008.

19. Vickers KC, Palmisano BT, Shoucri BM, Shamburek RD, Remaley AT. MicroRNAs are transported in plasma and delivered to recipient cells by high-density lipoproteins. Nat Cell Biol. 2011;13(4): 423-433.

20. Wang K, Zhang S, Weber J, Baxter D, Galas DJ. Export of microRNAs and microRNA-protective protein by mammalian cells. Nucleic Acids Res. 2010;38(2):7248-7259.

21. Mitchell PS, Parkin RK, Kroh EM, et al. Circulating microRNAs as stable blood-based markers for cancer detection. Proc Natl Acad Sci US A. 2008;105(30):10513-10518.

22. Sierzega M, Kaczor M, Kolodziejczyk P, Kulig J, Sanak M, Richter P. Evaluation of serum microRNA biomarkers for gastric cancer based on blood and tissue pools profiling: the importance of miR-21 and miR-331. Br J Cancer. 2017;117(2):266-273.

23. Fabris L, Ceder Y, Chinnaiyan AM, et al. The potential of microRNAs as prostate cancer biomarkers. Eur Urol. 2016;70(2):312-322.

24. Lawrie CH, Gal S, Dunlop HM, et al. Detection of elevated levels of tumour-associated microRNAs in serum of patients with diffuse large B-cell lymphoma. Br J Haematol. 2008;141(5):672-675.

25. Chen M, Calin GA, Meng QH. Circulating microRNAs as promising tumor biomarkers. Adv Clin Chem. 2014;67:189-214.

26. Bhattacharya S, Steele R, Shrivastava S, Chakraborty S, Di Bisceglie AM, Ray RB. Serum miR-30e and miR-223 as novel noninvasive biomarkers for hepatocellular carcinoma. Am J Pathol. 2016;186(2):242-247. 
27. Liu Y, Zhang G, Li H, et al. Serum microRNA-365 in combination with its target gene TTF-1 as a non-invasive prognostic marker for non-small cell lung cancer. Biomed Pharmacother. 2015;75:185-190.

28. Conev NV, Donev IS, Konsoulova-Kirova AA, Chervenkov TG, Kashlov JK, Ivanov KD. Serum expression levels of miR-17, miR-21, and miR-92 as potential biomarkers for recurrence after adjuvant chemotherapy in colon cancer patients. Biosci Trends. 2015;9(6):393-401.

29. Yoda Y, Takeshima H, Niwa T, et al. Integrated analysis of cancerrelated pathways affected by genetic and epigenetic alterations in gastric cancer. Gastric Cancer. 2015;18(1):65-76.

30. Qu Y, Zhang H, Duan J, et al. MiR-17-5p regulates cell proliferation and migration by targeting transforming growth factor-beta receptor 2 in gastric cancer. Oncotarget. 2016;7(22):33286-33296.

31. Yang L, Liang H, Wang Y, et al. MiRNA-203 suppresses tumor cell proliferation, migration and invasion by targeting Slug in gastric cancer. Protein Cell. 2016;7(5):383-387.

32. Ye G, Huang K, Yu J, et al. MicroRNA-647 Targets SRF-MYH9 axis to suppress invasion and metastasis of gastric cancer. Theranostics. 2017; 7(13):3338-3353.

33. Liu Z, Zhu J, Cao H, Ren H, Fang X. miR-10b promotes cell invasion through RhoC-AKT signaling pathway by targeting HOXD10 in gastric cancer. Int J Oncol. 2012;40(5):1553-1560.

34. Li Z, Lei H, Luo M, et al. DNA methylation downregulated mir-10b acts as a tumor suppressor in gastric cancer. Gastric Cancer. 2015;18(1):43-54.

35. Li X, Zhang Y, Shi Y, et al. MicroRNA-107, an oncogene microRNA that regulates tumour invasion and metastasis by targeting DICER1 in gastric cancer. J Cell Mol Med. 2011;15(9):1887-1895.

36. Wang $\mathrm{S}, \mathrm{Ma} \mathrm{G}, \mathrm{Zhu} \mathrm{H}$, et al. miR-107 regulates tumor progression by targeting NF1 in gastric cancer. Sci Rep. 2016;6:36531.

37. Feng L, Xie Y, Zhang H, Wu Y. miR-107 targets cyclin-dependent kinase 6 expression, induces cell cycle G1 arrest and inhibits invasion in gastric cancer cells. Med Oncol. 2012;29(2):856-863.

38. Selbach M, Schwanhausser B, Thierfelder N, Fang Z, Khanin R, Rajewsky N. Widespread changes in protein synthesis induced by microRNAs. Nature. 2008;455(7209):58-63.

39. Pradhan AK, Emdad L, Das SK, Sarkar D, Fisher PB. The enigma of miRNA regulation in cancer. Adv Cancer Res. 2017;135:25-52.

40. Tsujiura M, Ichikawa D, Komatsu S, et al. Circulating microRNAs in plasma of patients with gastric cancers. Br J Cancer. 2010;102(7): 1174-1179.

41. Zhang J, Song Y, Zhang C, et al. Circulating MiR-16-5p and MiR-19$\mathrm{b}-3 \mathrm{p}$ as two novel potential biomarkers to indicate progression of gastric cancer. Theranostics. 2015;5(7):733-745.

42. Tsai MM, Wang CS, Tsai CY, et al. Circulating microRNA-196a/b are novel biomarkers associated with metastatic gastric cancer. Eur J Cancer. 2016;64:137-148.

43. Hung PS, Chen CY, Chen WT, et al. miR-376c promotes carcinogenesis and serves as a plasma marker for gastric carcinoma. PLoS One. 2017 12(5):e0177346.

44. Hou CG, Luo XY, Li G. Diagnostic and prognostic value of serum MicroRNA-206 in patients with gastric cancer. Cell Physiol Biochem. 2016;39(4):1512-1520.

45. Zhu C, Ren C, Han J, et al. A five-microRNA panel in plasma was identified as potential biomarker for early detection of gastric cancer. Br J Cancer. 2014;110(9):2291-2299.

46. Huang Z, Zhu D, Wu L, et al. Six serum-based miRNAs as potential diagnostic biomarkers for gastric cancer. Cancer Epidemiol Biomarkers Prev. 2017;26(2):188-196.

47. Jensen SG, Lamy P, Rasmussen MH, et al. Evaluation of two commercial global miRNA expression profiling platforms for detection of less abundant miRNAs. BMC Genomics. 2011;12:435.

48. Cui L, Zhang X, Ye G, et al. Gastric juice microRNAs as potential biomarkers for the screening of gastric cancer. Cancer. 2013;119(9): 1618-1626.

49. Shao J, Fang PH, He B, et al. Downregulated microRNA-133a in gastric juice as a clinicopathological biomarker for gastric cancer screening. Asian Pac J Cancer Prev. 2016;17(5):2719-2722.
50. Zhang X, Cui L, Ye G, et al. Gastric juice microRNA-421 is a new biomarker for screening gastric cancer. Tumour Biol. 2012;33(6): 2349-2355.

51. Yu X, Luo L, Wu Y, et al. Gastric juice miR-129 as a potential biomarker for screening gastric cancer. Med Oncol. 2013;30(1):365.

52. Kao HW, Pan CY, Lai CH, et al. Urine miR-21-5p as a potential non-invasive biomarker for gastric cancer. Oncotarget. 2017;8(34): 56389-56397.

53. Valadi H, Ekstrom K, Bossios A, Sjöstrand M, Lee JJ, Lötvall JO. Exosome-mediated transfer of mRNAs and microRNAs is a novel mechanism of genetic exchange between cells. Nat Cell Biol. 2007; 9(6):654-659.

54. Ma M, Chen S, Liu Z, et al. miRNA-221 of exosomes originating from bone marrow mesenchymal stem cells promotes oncogenic activity in gastric cancer. Onco Targets Ther. 2017;10:4161-4171.

55. Wang N, Wang L, Yang Y, Gong L, Xiao B, Liu X. A serum exosomal microRNA panel as a potential biomarker test for gastric cancer. Biochem Biophys Res Commun. 2017;493(3):1322-1328.

56. Liu X, Zhang X, Zhang Z, et al. Plasma microRNA-based signatures to predict 3-year postoperative recurrence risk for stage II and III gastric cancer. Int J Cancer. 2017;141(10):2093-2102.

57. Zhang X, Yan Z, Zhang J, et al. Combination of hsa-miR-375 and hsamiR-142-5p as a predictor for recurrence risk in gastric cancer patients following surgical resection. Ann Oncol. 2011;22(10):2257-2266.

58. Park SK, Park YS, Ahn JY, et al. MiR 21-5p as a predictor of recurrence in young gastric cancer patients. J Gastroenterol Hepatol. 2016; 31(8):1429-1435.

59. Xue HG, Yang AH, Sun XG, Liu YY, Tian ZB. Expression of microRNA-328 functions as a biomarker for recurrence of early gastric cancer (EGC) after endoscopic submucosal dissection (ESD) by modulating CD44. Med Sci Monit. 2016;22:4779-4785.

60. Chu D, Zhao Z, Li Y, et al. Increased microRNA-630 expression in gastric cancer is associated with poor overall survival. PLoS One. 2014; 9(3):e90526

61. Tsai MM, Wang CS, Tsai CY, et al. MicroRNA-196a/-196b promote cell metastasis via negative regulation of radixin in human gastric cancer. Cancer Lett. 2014;351(2):222-231.

62. Gong J, Li J, Wang Y, et al. Characterization of microRNA-29 family expression and investigation of their mechanistic roles in gastric cancer. Carcinogenesis. 2014;35(2):497-506.

63. Komatsu S, Ichikawa D, Tsujiura M, et al. Prognostic impact of circulating miR-21 in the plasma of patients with gastric carcinoma. Anticancer Res. 2013;33(1):271-276.

64. Zhuang K, Han K, Tang H, et al. Up-regulation of plasma miR-23b is associated with poor prognosis of gastric cancer. Med Sci Monit. 2016; 22:356-361.

65. Yang Q, Zhang C, Huang B, et al. Downregulation of microRNA-206 is a potent prognostic marker for patients with gastric cancer. Eur $J$ Gastroenterol Hepatol. 2013;25(8):953-957.

66. Shi H, Han J, Yue S, Zhang T, Zhu W, Zhang D. Prognostic significance of combined microRNA-206 and CyclinD2 in gastric cancer patients after curative surgery: a retrospective cohort study. Biomed Pharmacother. 2015;71:210-215.

67. Ren C, Wang W, Han C, et al. Expression and prognostic value of miR-92a in patients with gastric cancer. Tumour Biol. 2016;37(7):9483-9491.

68. Zhang Y, Guan DH, Bi RX, Xie J, Yang CH, Jiang YH. Prognostic value of microRNAs in gastric cancer: a meta-analysis. Oncotarget. 2017;8(33):55489-55510.

69. Ding B, Gao X, Li H, Liu L, Hao X. A novel microRNA signature predicts survival in stomach adenocarcinoma. Oncotarget. 2017;8(17): 28144-28153.

70. Zheng B, Liang L, Wang C, et al. MicroRNA-148a suppresses tumor cell invasion and metastasis by downregulating ROCK1 in gastric cancer. Clin Cancer Res. 2011;17(24):7574-7583.

71. Sakamoto N, Naito Y, Oue N, et al. MicroRNA-148a is downregulated in gastric cancer, targets MMP7, and indicates tumor invasiveness and poor prognosis. Cancer Sci. 2014;105(2):236-243. 
72. Qiu X, Zhu H, Liu S, et al. Expression and prognostic value of microRNA-26a and microRNA-148a in gastric cancer. J Gastroenterol Hepatol. 2017;32(4):819-827.

73. Zhang Z, Sun J, Bai Z, et al. MicroRNA-153 acts as a prognostic marker in gastric cancer and its role in cell migration and invasion. Onco Targets Ther. 2015;8:357-364.

74. Chen W, Tang Z, Sun Y, et al. miRNA expression profile in primary gastric cancers and paired lymph node metastases indicates that miR-10a plays a role in metastasis from primary gastric cancer to lymph nodes. Exp Ther Med. 2012;3(2):351-356.

75. Ma G, Dai W, Sang A, Yang X, Gao C. Upregulation of microRNA-23a/b promotes tumor progression and confers poor prognosis in patients with gastric cancer. Int J Clin Exp Pathol. 2014;7(12):8833-8840.

76. Hashiguchi Y, Nishida N, Mimori K, et al. Down-regulation of miR$125 \mathrm{a}-3 \mathrm{p}$ in human gastric cancer and its clinicopathological significance. Int J Oncol. 2012;40(5):1477-1482.

77. Zhang Y, Wen X, Hu XL, Cheng LZ, Yu JY, Wei ZB. Downregulation of miR-145-5p correlates with poor prognosis in gastric cancer. Eur Rev Med Pharmacol Sci. 2016;20(14):3026-3030.

78. Li CY, Liang GY, Yao WZ, et al. Identification and functional characterization of microRNAs reveal a potential role in gastric cancer progression. Clin Transl Oncol. 2017;19(2):162-172.

79. Yang R, Fu Y, Zeng Y, et al. Serum miR-20a is a promising biomarker for gastric cancer. Biomed Rep. 2017;6(4):429-434.

80. Imaoka $\mathrm{H}$, Toiyama $\mathrm{Y}$, Okigami M, et al. Circulating microRNA-203 predicts metastases, early recurrence, and poor prognosis in human gastric cancer. Gastric Cancer. 2016;19(3):744-753.

81. Yuasa Y. Control of gut differentiation and intestinal-type gastric carcino genesis. Nat Rev Cancer. 2003;3(8):592-600.

82. Rotkrua P, Shimada S, Mogushi K, Akiyama Y, Tanaka H, Yuasa Y. Circulating microRNAs as biomarkers for early detection of diffusetype gastric cancer using a mouse model. Br J Cancer. 2013;108(4): 932-940.

83. Azarbarzin S, Feizi M, Safaralizadeh R, Kazemzadeh M, Fateh A. The value of MiR-383, an intronic miRNA, as a diagnostic and prognostic biomarker in intestinal-type gastric cancer. Biochem Genet. 2017;55(3): 244-252.

84. Xia L, Zhang D, Du R, et al. miR-15b and miR-16 modulate multidrug resistance by targeting BCL2 in human gastric cancer cells. Int J Cancer. 2008;123(2):372-379.

85. Li Q, Wang JX, He YQ, et al. MicroRNA-185 regulates chemotherapeutic sensitivity in gastric cancer by targeting apoptosis repressor with caspase recruitment domain. Cell Death Dis. 2014;5:e1197.
86. Korourian A, Roudi R, Shariftabrizi A, Madjd Z. MicroRNA-31 inhibits RhoA-mediated tumor invasion and chemotherapy resistance in MKN-45 gastric adenocarcinoma cells. Exp Biol Med (Maywood). 2017;242(18):1842-1847.

87. Kim CH, Kim HK, Rettig RL, et al. miRNA signature associated with outcome of gastric cancer patients following chemotherapy. BMC Med Genomics. 2011;4:79.

88. Smid D, Kulda V, Srbecka K, et al. Tissue microRNAs as predictive markers for gastric cancer patients undergoing palliative chemotherapy. Int J Oncol. 2016;48(6):2693-2703.

89. Shang Y, Feng B, Zhou L, et al. The miR27b-CCNG1-P53-miR-508-5p axis regulates multidrug resistance of gastric cancer. Oncotarget. 2016;7(1):538-549.

90. Sui M, Jiao A, Zhai H, et al. Upregulation of miR-125b is associated with poor prognosis and trastuzumab resistance in HER2-positive gastric cancer. Exp Ther Med. 2017;14(1):657-663.

91. Chen X, Liu XS, Liu HY, et al. Reduced expression of serum miR-204 predicts poor prognosis of gastric cancer. Genet Mol Res. 2016;15(2): 15027702 .

92. Su ZX, Zhao J, Rong ZH, Wu YG, Geng WM, Qin CK. Diagnostic and prognostic value of circulating miR-18a in the plasma of patients with gastric cancer. Tumour Biol. 2014;35(12):12119-12125. Retracted.

93. Tsujiura M, Komatsu S, Ichikawa D, et al. Circulating miR-18a in plasma contributes to cancer detection and monitoring in patients with gastric cancer. Gastric Cancer. 2015;18(2):271-279.

94. Liu S, Suo J, Wang C, et al. Prognostic significance of low miR-144 expression in gastric cancer. Cancer Biomark. 2017;20(4):547-552.

95. Guo X, Lv X, Lv X, Ma Y, Chen L, Chen Y. Circulating miR-21 serves as a serum biomarker for hepatocellular carcinoma and correlated with distant metastasis. Oncotarget. 2017;8(27):44050-44058.

96. Liu B, Su F, Chen M, et al. Serum miR-21 and miR-125b as markers predicting neoadjuvant chemotherapy response and prognosis in stage II/III breast cancer. Hum Pathol. 2017;64:44-52.

97. Witwer KW. Circulating microRNA biomarker studies: pitfalls and potential solutions. Clin Chem. 2015;61(1):56-63.

98. Tiberio P, Callari M, Angeloni V, Daidone AG, Appierto V. Challenges in using circulating miRNAs as cancer biomarkers. Biomed Res Int. 2015;2015:731479.
OncoTargets and Therapy

\section{Publish your work in this journal}

OncoTargets and Therapy is an international, peer-reviewed, open access journal focusing on the pathological basis of all cancers, potential targets for therapy and treatment protocols employed to improve the management of cancer patients. The journal also focuses on the impact of management programs and new therapeutic agents and protocols on

\section{Dovepress}

patient perspectives such as quality of life, adherence and satisfaction The manuscript management system is completely online and includes a very quick and fair peer-review system, which is all easy to use. Visit http://www.dovepress.com/testimonials.php to read real quotes from published authors. 